\title{
Numerische Untersuchung von Rohrströmungen am Beispiel eines Bifurkators
}

\begin{abstract}
Zusammenfassung: Im Beitrag wird die hydraulische Situation eines Bifurkators numerisch berechnet. Dieser, ausgeführt als Hosenrohr, wurde in einem früheren Modellversuch im Wasserbaulabor der TU-Graz untersucht. Die Ergebnisse des Modellversuchs werden zur Kalibrierung der numerischen Berechnung verwendet. Durch die gute Übereinstimmung des Modellversuches mit der numerischen Berechnung wird gezeigt, dass numerische Rechenprogramme ein leistungsfähiges, wichtiges Werkzeug für hydraulische Untersuchungen darstellen.
\end{abstract}

Numerical analysis of a bifurcation piece to demonstrate flow in tubes

Summary: The hydraulic situation in a bifurcation piece has been calculated by numerical means. This piece, which is designed as a Y-branch pipe, was studied in an earlier hydraulic scale model test at the hydraulics laboratory of the Graz University of Technology. The results of the test have been used to calibrate the numerical analysis. The good agreement between the results of model test and the numerical analysis demonstrates that numerical calculation programs are an important and efficient tool of hydraulic analysis.

\section{Einleitung}

Numerische Rechenprogramme (CFD-Programme) finden in der Planung und Konzeption wasserbaulicher Anlagen zunehmend praktische Anwendung und stellen ein effektives sowie effizientes Werkzeug zur Untersuchung hydraulischer Aufgabenstellungen dar. Wegen der komplexen mathematischen - physikalischen Zusammenhänge bedarf es sowohl besonderer Sorgfalt bei der Anwendung numerischer Verfahren als auch bei der Interpretation der berechneten Ergebnisse. Aufgrund der ständigen Verbesserung und Weiterentwicklung der numerischen Modelle mit ihren theoretischen und semi-empirischen Ansätzen kommt den Ergebnissen aus physikalischen Modellversuchen - früheren sowie laufenden - eine wichtige Be-

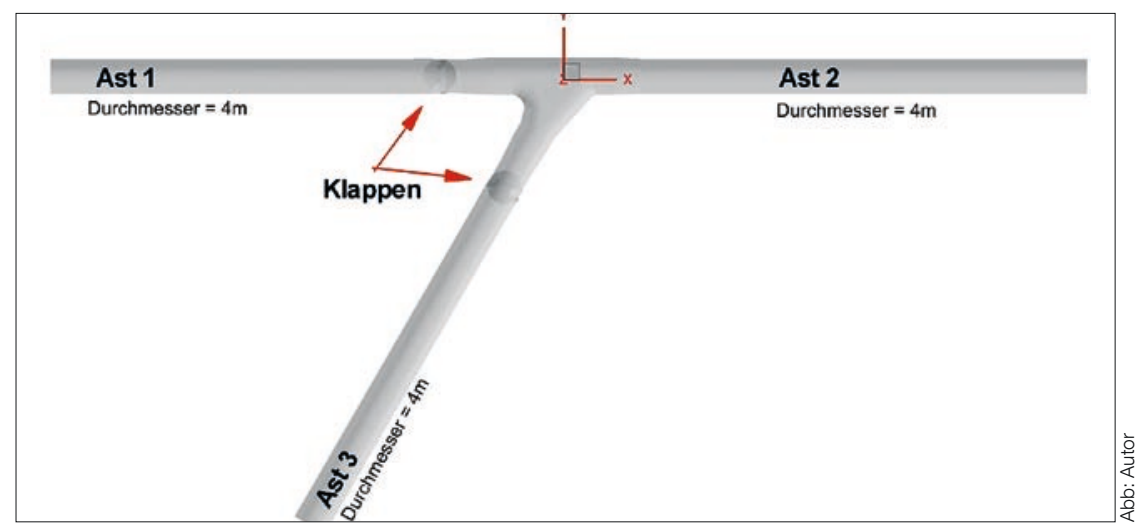

Abb. 1: Geometrie des Abzweigers, Grundriss.

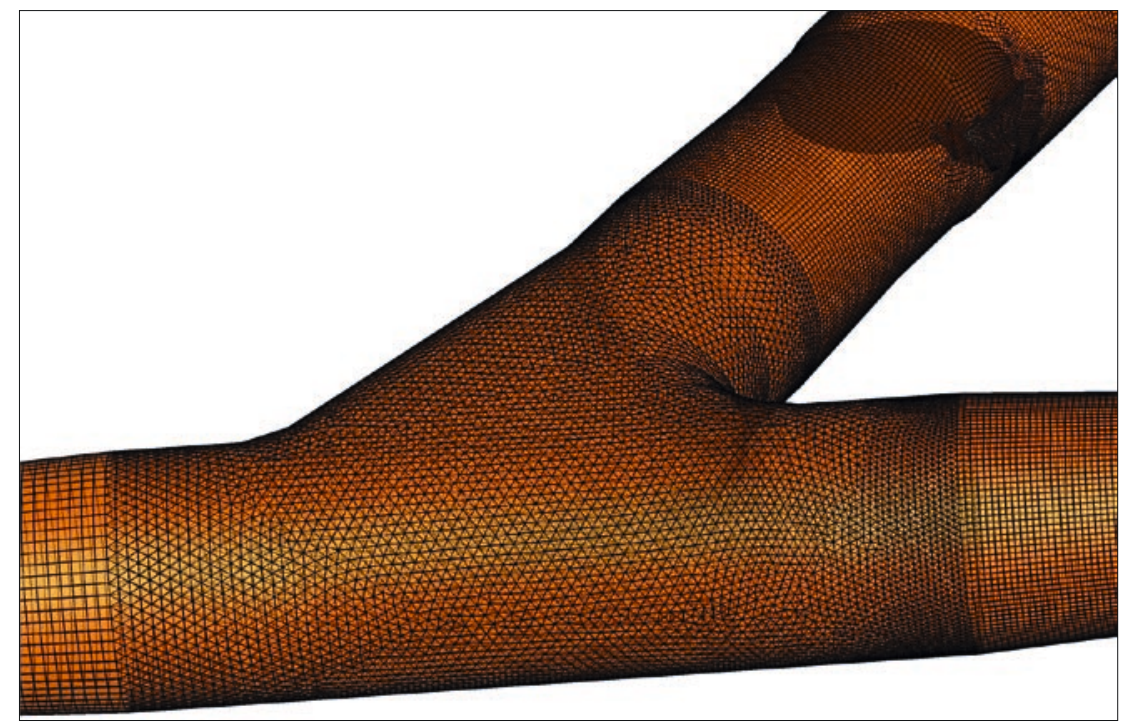

Abb. 2: Detailansicht des Netzes.

deutung zu. Ist ein numerisches Modell im Hinblick auf die Diskretisierung und das Rechenverfahren kalibriert und validiert, erlaubt dieses eine kostengünstige und schnelle Durchführung sowohl von Variantenstudien (z.B. Untersuchung verschiedener Lastfälle) als auch einer Projekts- oder Bauteilsoptimierung. Darüber hinaus können Berechnungen für sehr aufwändige Systeme durchgeführt und sehr anschaulich und aussagekräftig ausgewertet werden.

\section{Aufgabenstellung}

Anhand eines Beispiels für den Triebwasserweg einer Hochdruckanlage werden die Ergebnisse einer 3-D-numerischen Berechnung den bereits vorliegenden Versuchsergebnissen aus einem physikalischen Modellversuch gegenüber gestellt.

Der interessierende Untersuchungsbereich dieses Triebwasserweges bildet ein Rohrabzweigstück (Hosenrohr) mit zwei Absperrklappen für das die Strömungsverhältnisse für unterschiedliche Lastfälle zu betrachten sind. Das Hosenrohr zusammen mit den zwei Absperrklappen und den erforderlichen Beruhigungsstrecken werden im Modell berücksichtigt und die auftretenden Druckverteilungen im Hosenrohr mit Hilfe einer numerischen Berechnung quantifiziert. 


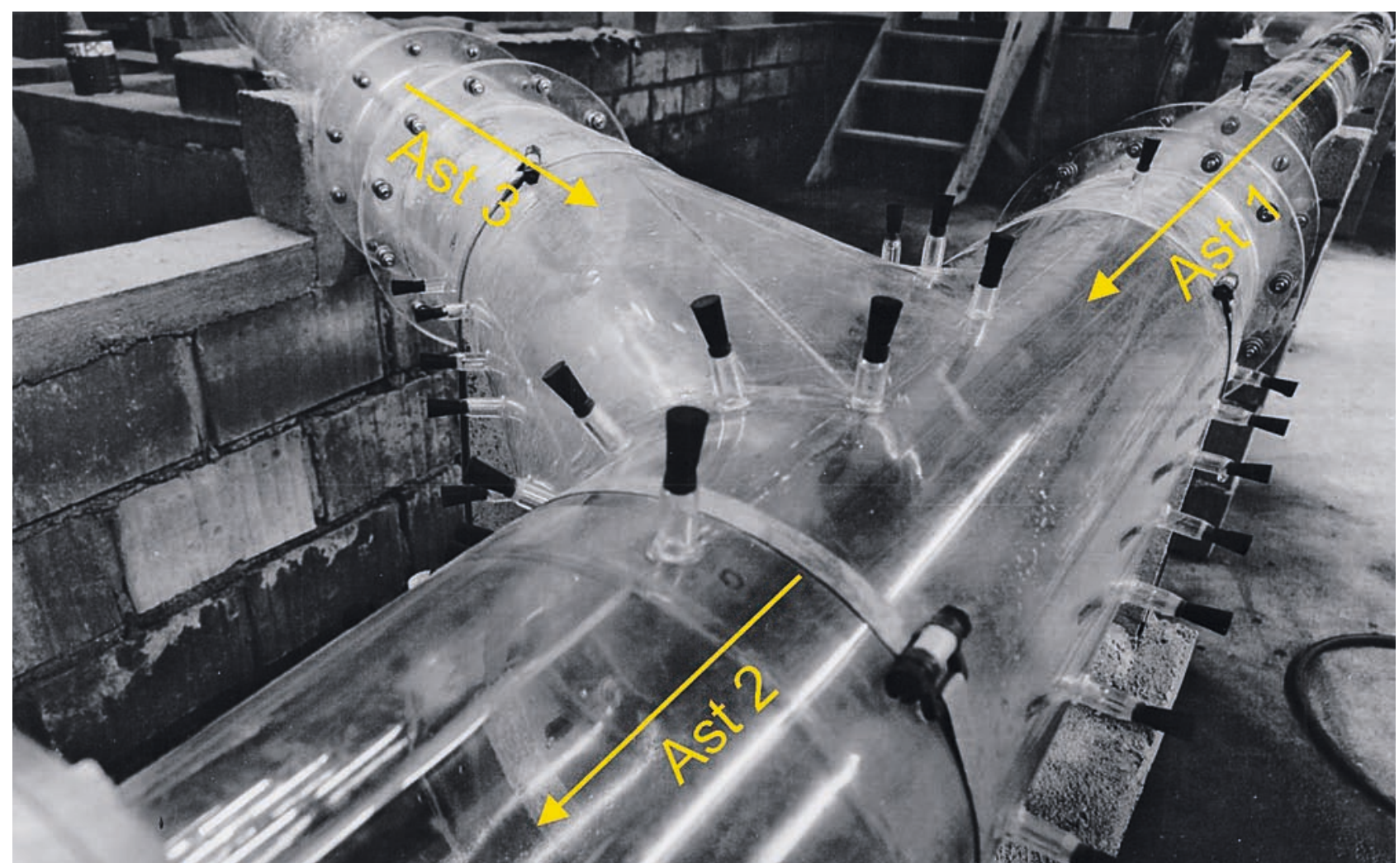

Abb. 4: Modellversuch eines Abzweigers an der TU-Graz.

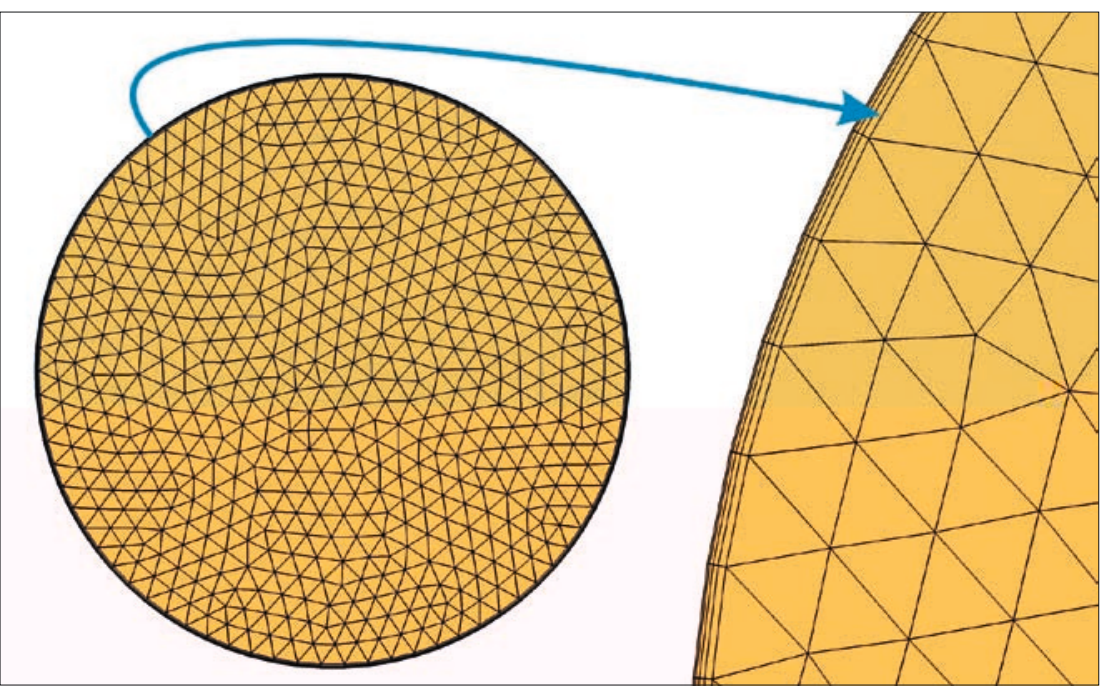

Abb. 3: Netzverdichtung in Wandnähe.
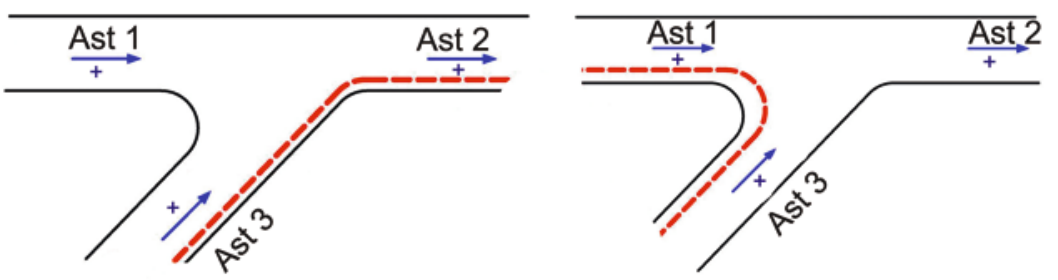

Abb. 5: Druckmessung im Modell, rote Line kennzeichnet die Stelle für die Druckmessung in der Symmetrieebene. Positive Fließrichtung mit blauen Pfeilen definiert.

\section{Numerisches Modell -}

Diskretisierung und Geometrie

Als ersten Schritt wird die Geometrie des Modells aus vorhandenen Konstruktionsplänen erstellt und anschließend diskretisiert, d.h. das Volumen wird mit der Software Gambit (Version 2.4.6) in finite Elemente unterteilt ( $A b b .1$ und $A b b .2$ ). Da das verwendete numerische Rechenprogramm (Fluent 6.3.26) auf der FinitenVolumen-Methode basiert (Fluent, 2005), werden an diesen Elementen die Bilanzgleichungen (Massentransport, Impulserhaltung) aufgestellt und numerisch gelöst.

Bei der Netzerstellung werden Hexaeder-Elemente angestrebt, da diese im Vergleich zu Tetraeder-Elementen die Anzahl der erforderlichen Elemente und somit die erforderliche Rechenzeit verringert. Wie in Abbildung 2 ersichtlich, ist es nicht immer möglich, Hexaeder-Elemente zu verwenden, da eine komplizierte Geometrie mit ihren vielen Verschneidungen und gekrümmten Flächen, zumeist die Verwendung von TetraederElementen erfordert.

Um den starken Geschwindigkeitsgradient in Wandnähe in der numerischen Berechnung richtig abzubilden ist eine Netzverdichtung bis in die viskose Unterschicht durchzuführen. Damit aber die 


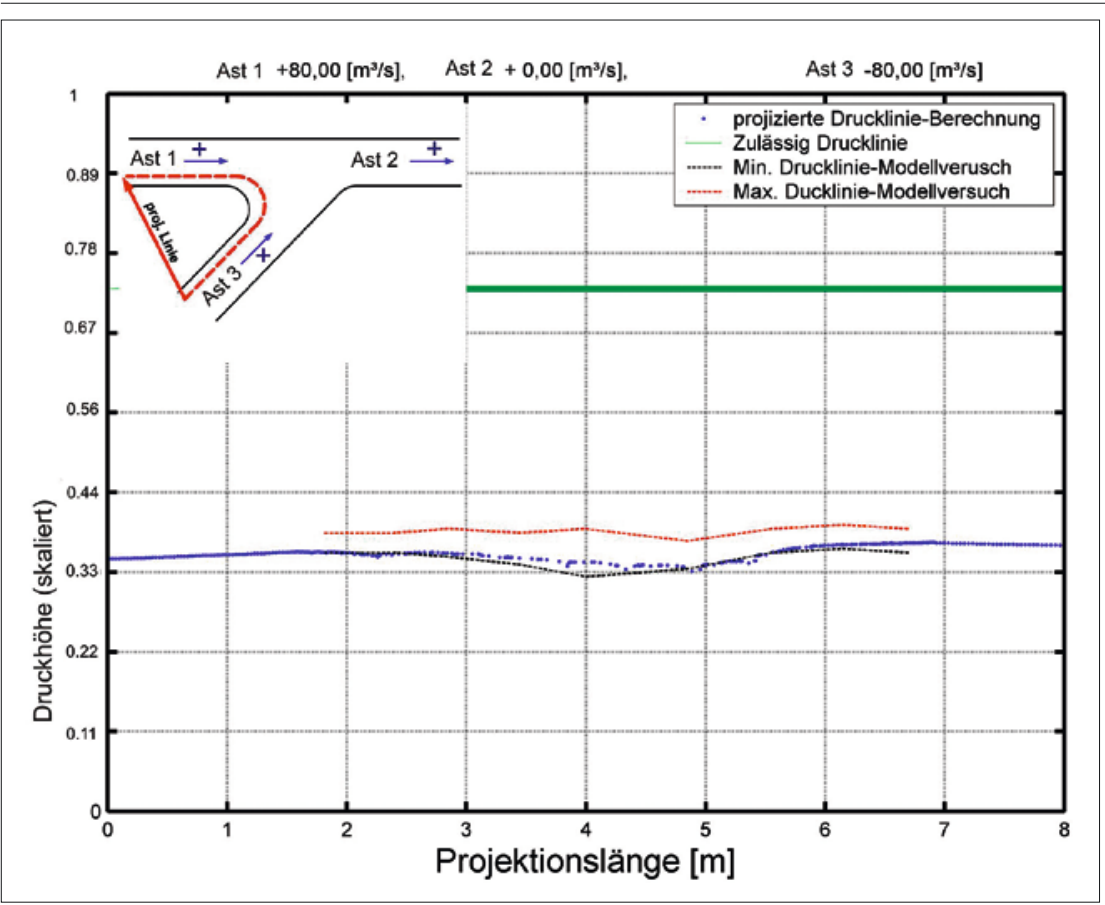

Abb. 6: Teilergebnis der Kalibrierung. Blaue Linie ist das Ergebnis der numerischen Berechnung. Rote und schwarze Linie ist das Ergebnis des Modellversuchs (Druckhöhe skaliert).

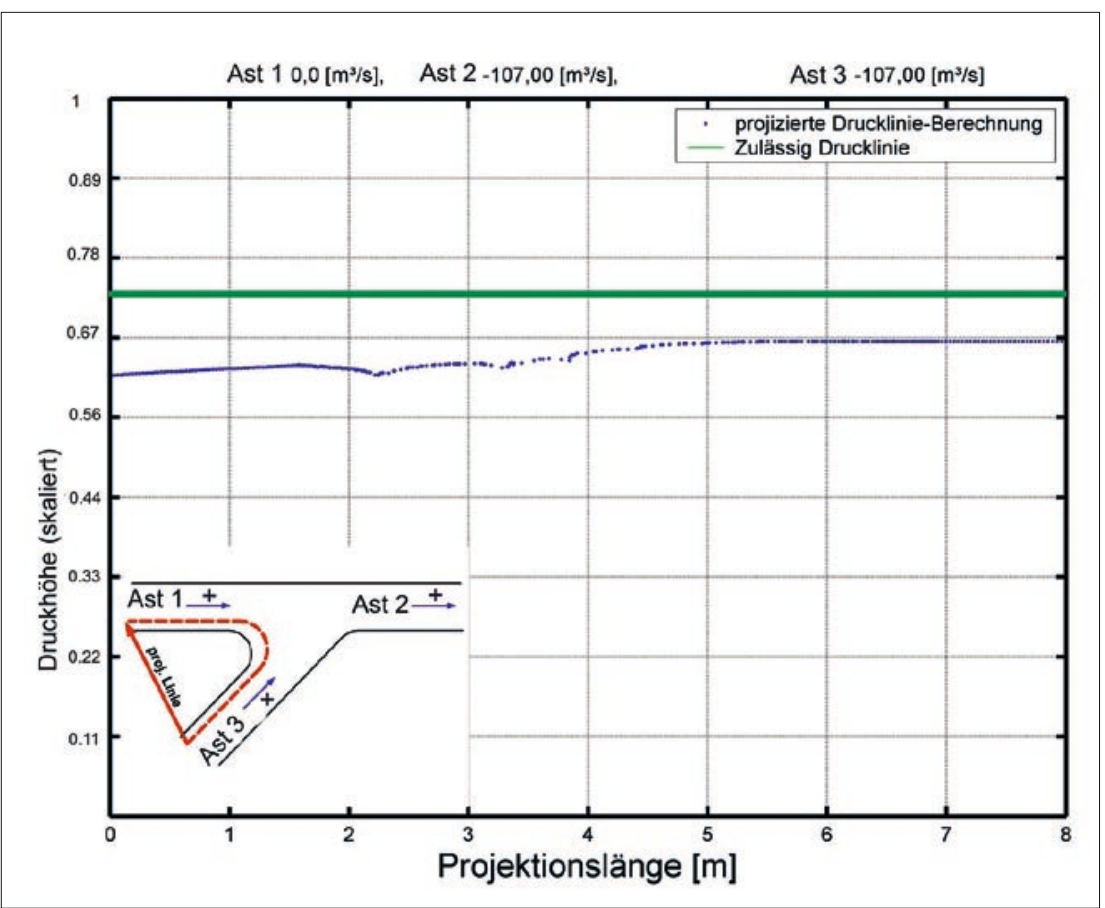

Abb. 7: Ergebnis eines Lastfalls mit erhöhtem Durchfluss. Blaue Line ist das Ergebnis der numerischen Berechnung. Grüne Line ist der maximale zulässige Druck (Druckhöhe skaliert).

Elementanzahl und damit die Rechenzeit nicht zu groß werden, wird das logarithmische Wandmodell angewendet womit eine Auflösung der viskosen Unterschicht nicht mehr notwendig ist.

Der Wandabstand für die ersten Elemente in der Grenzschicht muss im Bereich des dimensionslosen Wandabstand $\mathrm{y}^{+} \mathrm{zwi}-$ schen 30 bis 300 liegen. $\mathrm{y}^{+}$hat die Form einer Reynoldszahl und lautet folgend:

$$
y^{+}=\frac{y u_{\tau}}{v} \quad[-]
$$

Dabei ist $u_{\tau}$ die Schubspannungsgeschwindigkeit, $v$ die kinematische Viskosität und $y$ der Wandabstand.
Ein zweites wichtiges Merkmal neben dem dimensionslosen Wandabstand $\mathrm{y}^{+}$ ist die Unabhängigkeit der numerischen Lösung von der Anzahl der Elemente, die die Feinheit des Netzes darstellen. Dabei wird die numerische Berechnung mit denselben Randbedingungen mit mehreren verschieden feinen Netzen berechnet. Wenn die numerische Lösung konvergiert, das heißt, dass sich auch bei weiterer Verfeinerung des Netzes nur noch unwesentliche Änderungen im Ergebnis auftreten, kann dieses Netz für die eigentliche Berechnung dann verwendet werden.

Nachdem das Netz des Abzweigers und deren Randbedingungen (z. B. Rauigkeit, Durchflüsse) definiert sind, erfolgt im nächsten Schritt die Kalibrierung.

\subsection{Kalibrierung der numerischen Berechnung}

Im Wasserbaulabor der Technischen Universität Graz ist der Abzweiger in einem physikalischen Modellversuch untersucht worden.

In diesem Modellversuch sind Druckmessungen an ausgezeichneten Stellen durchgeführt. Beispielhaft sind in Abbildung 5 zwei Bereiche dargestellt an denen im Modell die Druckhöhen punktuell gemessen werden.

Ein Teilergebnis der Kalibrierung ist in Abbildung 6 ersichtlich. Die Strömungsrichtung ist von Ast 1 nach Ast 3 mit einem Volumenstrom von $80\left[\mathrm{~m}^{3} / \mathrm{s}\right]$ angegeben, d.h. Ast 2 wird nicht durchströmt. Die rote sowie schwarze Linie stellen die minimalen und maximalen Drückhöhen aus dem physikalischen Modellversuch dar.

Die blaue Linie zeigt das Ergebnis der numerischen Berechnung, welche sehr gut innerhalb der Bandbreite des Modellversuchs (Bereich zwischen roter und schwarzer Linie) zu liegen kommt. Durch das Ergebnis der Kalibrierung, wo Modellparameter wie Rauigkeit oder Turbulenzmodelle verändert wurden, ist somit gewährleistet, dass für jene Lastfälle, für welche es keine Druckmessungen aus dem Modellversuch gibt, entsprechend verlässliche Ergebnisse berechnet werden können.

\section{Berechnungsergebnisse}

Ein Beispiel eines untersuchten Lastfalls aus der numerischen Berechnung, für den keine vergleichbaren Modellergebnisse 


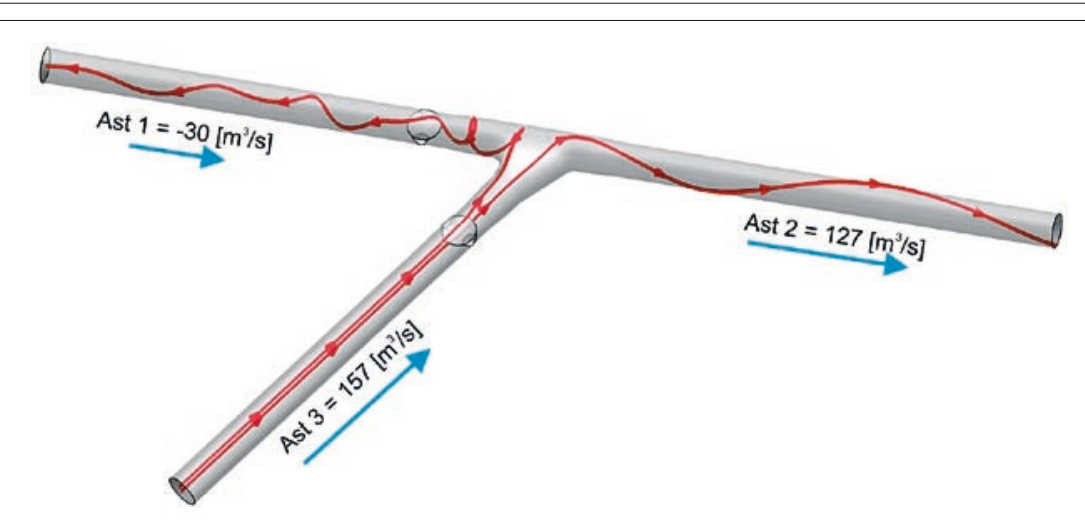

Abb. 8: Darstellung der numerischen Berechnung - Stromlinien.

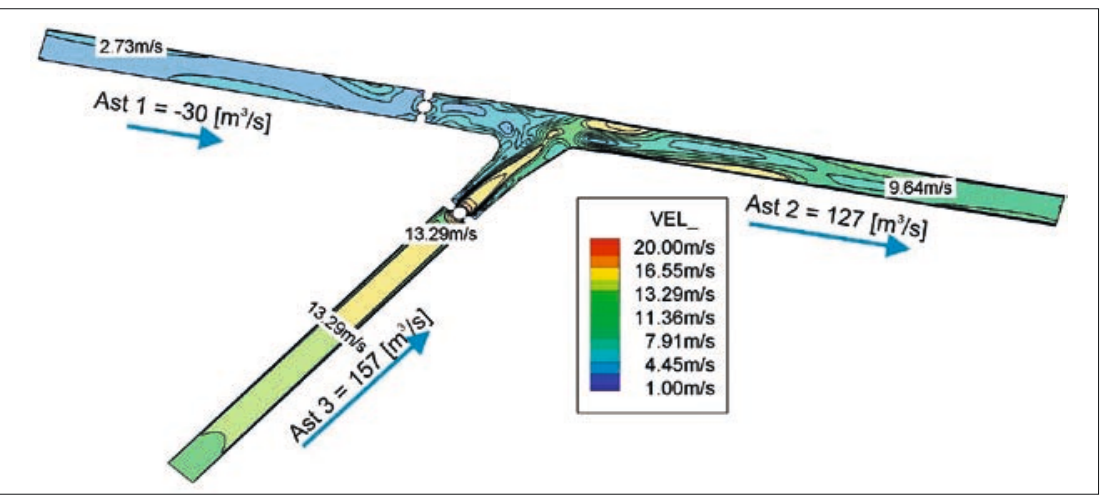

Abb. 9: Geschwindigkeitsverteilung in horizontaler Systemebene.

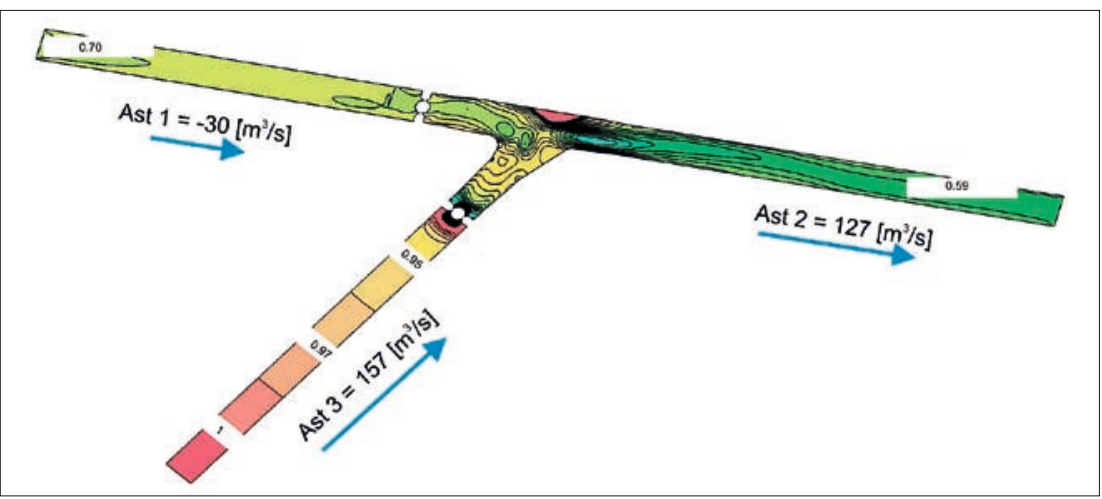

Abb. 10: Druckverteilung (mit maximalem Druck skaliert) in horizontaler Systemebene.

vorliegen, ist in Abbildung 7 dargestellt. Durch den im Vergleich zur Kalibrierung erhöhten Durchfluss (Ast 2 und Ast 3 mit $\left.107\left[\mathrm{~m}^{3} / \mathrm{s}\right]\right)$ ergibt sich nach der numerischen Berechnung auch eine höhere Drucklinie im Abzweiger. Dieses Druckniveau, das durch die blaue Linie in Abbildung 7 dargestellt ist, liegt wesentlich näher an der maximalen zulässigen Drucklinie (Linie grün). Damit konnte gezeigt werden, dass - bei entsprechender Steigerung des Durchflusses - an keinem wesentlichen und interessierenden Punkt im Abzweiger eine Überschreitung des zulässigen Drucks zu erwarten ist.

\subsection{Detailauswertung der numerischen Berechnung}

Aufgrund der numerischen Lösung für das gesamte betrachtete Gebiet, besteht die Möglichkeit, die Berechnungsergebnisse sehr anschaulich und detailliert darzustellen. Zur Visualisierung von Strömungsverläufen dienen dazu beispielsweise Stromlinien (siehe $A b b$. 8, Durchfluss: Ast $1=-30$, Ast $2=127$ und Ast $3=157\left[\mathrm{~m}^{3} / \mathrm{s}\right]$ ). Daraus sind sehr klar die Sekundärströmungseffekte zu erkennen, die sich aufgrund der unsymmetrischen Stromtrennung im Abzweiger einstellen. In Abbildung 10 ist die
Druckverteilung des Abzweigers dargestellt, welche bis zur Absperrklappe einen linearen Druckabfall von 1 auf 0,95 [-] aufweist (Werte sind mit dem maximalen Druck skaliert). Aufgrund von Strömungsablösungen und daraus resultierender Wirbelbildung im Bereich der Klappe verändert sich dieser lineare Druckabfall und es kommt zu einem verstärkten Druckverlust. Dies erlaubt die Berechnung des dimensionslosen Verlustbeiwertes $\zeta$ für diese Anlagenkonfiguration und kann im Weiteren für die Berechnung des gesamten hydraulischen Systems herangezogen werden. Ein ähnliches Bild zeigen auch die Geschwindigkeitsverteilungen ( $A b b .9$ ) im Abzweiger: Zuerst ist eine voll ausgebildete turbulente Rohrströmung mit der charakteristischen Geschwindigkeitsverteilung vorhanden, die dann durch den Abzweiger aufgehoben wird, es kommt dann zu einer ausgeprägten Sekundärströmung.

\section{Zusammenfassung}

Die numerische Simulation der Rohrhydraulik stellt eine sehr effektive Alternative zum physikalischen Modellversuch dar, womit die Ergebnisse rasch ermittelt werden können. Auf die Wichtigkeit der Kalibrierung und Validierung von Rechenergebnissen ist besonders hinzuweisen, da durch z.B. beschränkte Ressourcen (Prozessorleistung für eine geeignet feine Diskretisierung) falsche Ergebnisse berechnet werden

Geänderte Rahmenbedingungen durch einen liberalisierten Strommarkt erfordern auch eine geänderte Einsatzweise und Betriebsführung bestehender Wasserkraftanlagen. Für eine Überprüfung der hydraulischen Funktionsfähigkeit von Anlageteilen unter geänderten Voraussetzungen stellen numerische Rechenverfahren somit ein effizientes und wertvolles Werkzeug dar.

Korrespondenz:

DI Wolfgang Doble

Graz University of Technology

Institute of Hydraulic Engineering and

Water Resources Management

Stremayrgasse 10/l

8010 Graz

E-Mail: w.dobler@tugraz.at

\section{LITERATUR}

Fluent (2005) Fluent 6.2 User's Guid. Fluent Inc. Mayr D (2002) Hydraulische Untersuchungen An Dreifach-Rohrverzweigungen (Trifurcatoren),

Schriftenreihe zur Wasserwirtschaft TU-Graz.

ISBN 978-3-85125-066-4. DownloadLink: http:// portal.tugraz.at/portal/page/portal/TU_Graz/Einrichtungen/Institute/Homepages/i2130/links/ links_veroeffentlichungen 